\title{
Propagating speed waves in flocks: A mathematical model
}

\author{
Andrea Cavagna, ${ }^{1}$ Daniele Conti,${ }^{1,2,3,{ }^{*}}$ Irene Giardina, ${ }^{1,2,4}$ and Tomas S. Grigera ${ }^{5,6}$ \\ ${ }^{1}$ Istituto Sistemi Complessi, Consiglio Nazionale delle Ricerche, UOS Sapienza, 00185 Rome, Italy \\ ${ }^{2}$ Dipartimento di Fisica, Università Sapienza, 00185 Rome, Italy \\ ${ }^{3}$ Laboratoire de physique statistique, CNRS, UPMC and École normale supérieure, 75005 Paris, France \\ ${ }^{4}$ INFN, Unità di Roma 1, 00185 Rome, Italy \\ ${ }^{5}$ Instituto de Física de Líquidos y Sistemas Biológicos CONICET, Universidad Nacional de La Plata, La Plata, Argentina \\ ${ }^{6}$ CCT CONICET La Plata, Consejo Nacional de Investigaciones Científicas y Técnicas, La Plata, Argentina
}

(Received 17 November 2017; revised manuscript received 18 June 2018; published 8 November 2018)

\begin{abstract}
An efficient collective response to external perturbations is one of the most striking abilities of a biological system. One of the crucial aspect of this phenomenon is given by the information transfer, and resulting propagation of signals, within the group. In this respect the existence of density waves that propagate linearly on a flock of birds is well known. However, most aspects of this phenomenon are still not fully captured by theoretical models. In this work we present a model for the propagation of speed fluctuations inside a flock, which is able to reproduce the observed density waves. We study the full solution of the model in $d=1$, and we find a line in the parameter space along which the system relaxes as fast as possible without oscillating, resembling a generalized critical damping condition. By analyzing the parameters plane we show that this "critical damping" line indeed represents an attractor for a steepest descent dynamics of the return time of the system. Finally we propose a method to test the validity of the model through future experiments.
\end{abstract}

DOI: 10.1103/PhysRevE.98.052404

\section{INTRODUCTION}

Many intriguing phenomena of the living world arise from the interactions between the components of a biological system. When a group of interacting individuals behave very differently compared to a collection of the same, but noninteracting, individuals, one usually speaks of collective behavior [1-5]. In physics, the emergence of collective behavior has been extensively investigated. Statistical mechanics proved to be a very powerful theory for understanding how macroscopic phenomena arise from the interaction of many microscopic components [1-3]. This success has reinforced the belief that it might be possible to use the same concepts and mathematical apparatus for describing the collective properties of biological systems. In addition, due to the technological progress of recent years, emergent biological phenomena are now susceptible to quantitative large-scale experiments, motivating the opening up of new challenges at the interface between physics and biology [6,7].

An interesting feature of collective behavior in biological systems lies in the efficiency of the system's response to stimuli coming from the external environment. Understanding how a group is able to respond as a whole also has important consequences for technological developments and control theory [8-11]. An efficient collective response evidently requires that the system react quickly, but it is also important that the system does not take too much time to return to its unperturbed state. In order to transmit a signal across the group, each individual must be displaced from its

\footnotetext{
*Corresponding author: daniele.conti@uniroma1.it, dconti@lps.ens.fr
}

original state (which is not necessarily a rest state), but then the question arises: how will it go back to its original state? The answer depends on the balance between transmitting the signal in the quickest way and disrupting as little as possible the state of the system. Here we investigate this problem.

Collective response is an ability shared by many biological systems at different scales: bird flocks [12], swarms of insects [13], herds of mammals [14], bacterial clusters [15,16], cells [17], fish schools [18,19], marching locusts [20], ant trails [21], etc.; even at the human social level it is possible to find such features [22,23]. Among biological systems, flocks of birds have attracted much attention and have been studied both theoretically and experimentally [12,24-27]. In particular in Refs. [28-31] the authors highlight the importance of information propagation for the achieving of an efficient collective response in flocks: in order for the group to respond to a perturbation felt only by some individuals, it is necessary that information flows within the group [32]. A vivid manifestation of propagation phenomena is the occurrence of density waves: video observations on large flocks of starlings show the formation of waves in proximity of an arriving predator and their propagation through the group [30].

The origin of density waves could be due to different mechanisms. Models of collective motion and polar active systems [5,33] display anomalous density fluctuations [34] due to the nontrivial coupling between directional and positional degrees of freedom in these systems. In the ordered phase this coupling gives rise to density waves on the very large scale, which have been studied using a hydrodynamic approach [26,35-42]. While these hydrodynamic density waves (HDWs) are certainly relevant for a variety of active systems, it is not clear whether they fully account for what is observed in natural flocks, for the following reasons: (1) HDWs relate 
to the essence of the hydrodynamic approach, which considers the limit $L, t \rightarrow \infty$, while natural systems are often far from these limits and exhibit important collective phenomena over medium scales; (2) HDWs have an anisotropic propagation not shown by experimental observations: in the reference frame of the flock they propagate mainly in the direction orthogonal to the motion of the flock (suppressing longitudinal modes) ${ }^{1}$ [26], while experiments suggest that what matters is the direction of the arriving predator; (3) HDWs are derived only from the fluctuations in the orientations and are in fact coupled to these $[26,35,36]$.

There are other possible mechanisms that generate density waves besides HDWs. As highlighted, for example, in Refs. [43-45], we can consider fluctuations not only in the orientations of the velocity but also in the speed. Empirical data show that fluctuations in the speed are long-range, that is, the correlation is scale-free. To reproduce such correlations one needs to explicitly allow for speed variability in the individual equation of motion $[43,44,46]$. In this context, it is reasonable to hypothesize that density waves have a contribution also coming from the speed.

In this work we present a model of collective motion in flocks of birds that admits the linear propagation of fluctuations in the individual speed of flight. To do this, we follow a similar theoretical path to the one used for describing turning waves [29]. We derive a dynamic equation for the speed that turns out to have an analogous structure as the telegraph equation, i.e., the equation that describes the propagation of electromagnetic waves in telegraph cables [47-49]. This equation has a special point in the space of parameters in which it assumes a simpler form, very similar to that of a pure wave equation. We highlight this interesting aspect by analyzing the dispersion relation and by making an analogy with critical damping. Then we confirm the presence of a generalized critical damping mechanism by studying the full solution of the equation in $d=1$. This last feature addresses the question raised above: minimizing the return time to the steady state improves the efficiency of the collective response. Finally we suggest a method by which one can experimentally verify the fundamental hypotheses of the model.

\section{A NEW EQUATION OF MOTION FOR THE SPEED}

We look for a simple mathematical model able to reproduce a generalized wave equation for the individual speed $v_{i}=\left|\mathbf{v}_{i}\right|$.

\footnotetext{
${ }^{1}$ There are two main reason why they are suppressed. The first one concerns the fact that, as observed in Ref. [34], longitudinal traveling bands move with the same velocity of the flock. This means that in the reference frame of the flocks they are not propagating. The second reason is that bands become less sharp, and eventually disappear, as the noise amplitude is decreased away from the transition point. Therefore traveling bands are a phenomenon that happens close to the critical point, for high values of the noise, while in the case of flocks we are in the deeply ordered phase, which is reached for low values of the noise. As a consequence in this regime there are no traveling bands.
}

\section{A. From speed waves to density waves}

The reader may well ask why we want to introduce speed waves in order to generate density waves. First, we note that in biological active matter, and in particular in flocks, one of the most important quantities used to study a system is the correlation of the velocities. As a consequence the velocity (and hence the speed) is one of the fundamental degrees of freedom with which a system is described. Velocity fluctuations in starling flocks are correlated over long distances, and this applies to fluctuations of speed as well as direction. Long-range-correlated fluctuations of speed occur only for values of the control parameter very near a specific value (i.e., in the close vicinity of a critical point). The question arises: is there some usefulness to long-range correlations that can justify this fine-tuning of the system parameters? It is possible to argue that a scale-free correlation function is a necessary condition (not sufficient, though) to transfer information across the group without the need of encoding or decoding, independently of the group's size [50]. Hence, strong correlation seems functional to having an effective information transfer, suggesting that speed fluctuations are finetuned to achieve information propagation of speed variations, although up to now only transmission of orientation variations has been unambiguously observed. That said, we can presume to link the presence of speed waves to the creation of density waves. To see this simply, let us put ourselves in $d=1$ : in this case there are no directional degrees of freedom (orientations), then, for the generation of density waves, other sources are needed. Let us suppose that speed fluctuations $u(x, t)$ already satisfy a wave equation, that is, $u(x, t)=f(x-c t)$. Now all we need is just to recall the linearized continuity equation:

$$
\frac{\partial \delta \rho}{\partial t}+\rho_{0} \frac{\partial u}{\partial x}=0
$$

where $\delta \rho$ is the density fluctuation and $\rho_{0}$ the mean component. At this point we note that in order to satisfy the continuity equation with a propagating speed fluctuations, it is sufficient that the density also satisfies a wave equation: $\delta \rho(x, t)=\frac{\rho_{0}}{c} f(x-c t)$.

\section{B. Velocity alignment and pseudo-Hamiltonian description}

The starting point of any model of collective motion is some kind of interaction between the components of the system $[25,33,46,51-55]$. Here we will consider as the fundamental interaction the tendency of each particle to align its velocity with those of its neighbors. We can describe the dynamics of the system with a general stochastic equation of motion for the positions $\mathbf{r}_{i}$ and velocities $\mathbf{v}_{i}$ of $N$ interacting individuals $(i=1, \ldots, N)$

$$
\begin{aligned}
\frac{d \mathbf{r}_{i}}{d t} & =\mathbf{v}_{i}, \\
\frac{d \mathbf{v}_{i}}{d t} & =\mathbf{F}_{i}+\zeta_{i},
\end{aligned}
$$

where $\mathbf{F}_{i}$ is an effective force vector encapsulating the effects due to the other particles $j \neq i$ and $\zeta_{i}$ is a stochastic force described by a random white noise $\left\langle\zeta_{i}(t) \zeta_{j}\left(t^{\prime}\right)\right\rangle=2 \eta T \delta_{i j} \delta(t-$ $t^{\prime}$ ), where $T$ is a generalized temperature that allows us to tune 
the magnitude of the noise. Since we want an imitation rule at the base of the model, we can describe $\mathbf{F}_{i}$ as a social force

$$
\mathbf{F}_{i}=J \sum_{j \neq i} n_{i j} \mathbf{v}_{j}
$$

where, for the sake of generality and for future convenience, we introduced the parameter $J$ setting the scale of such force, while the matrix $n_{i j}$ is the connectivity matrix, which defines the neighborhood of interaction of $i$ (metric [33,56,57] or topological [58-60]). We note that the social force can be written as $F_{i}=-\delta \mathcal{H} / \delta \phi_{i}$, where

$$
\mathcal{H}=-\frac{J}{2} \sum_{i, j} n_{i j} \mathbf{v}_{i}(t) \cdot \mathbf{v}_{j}(t) .
$$

It is tempting to interpret $\mathcal{H}$ as a Hamiltonian for a dissipative Langevin dynamics, but, due to the active nature of the system, we have to be careful [61]. Active matter systems are out of equilibrium, the constituents absorb and dissipate energy, and therefore detailed balance is not valid. As a consequence, the stationary probability distribution is not given by the Boltzmann weight $P(\{\mathbf{v}\}) \neq e^{-\beta \mathcal{H}\{\mathbf{v}\}} .^{2}$

The activity of a system of self-propelled individuals comes from the rearranging of the interaction network: animals move relative to each other, changing neighbors over time [65]. Actually the connectivity matrix depends on time through the positions $\mathbf{r}_{i}(t)$, which change with the velocities $\mathbf{v}_{i}(t)$, so that $n_{i j}=n_{i j}(t)$. The hydrodynamic theory of Toner and $\mathrm{Tu}[26,35]$ takes into account this aspect providing a continuous description of a coarse-grained velocity field $\mathbf{v}(\mathbf{x})$ where the movement of the network is fully included through the introduction of a coarse-grained density field $\rho(\mathbf{x})$. However, since we are interested in characterizing fast information propagation across finite-size systems, an hydrodynamic description, relying on asymptotically long times and very long distances, is not suitable. When considering long times, the rearrangement of the network cannot be overlooked; however, experimental data show that the local rearrangement of the network in natural flocks happens on timescales much larger than the local updating time of the velocities [65]. As a consequence birds are in a state of local quasiequilibrium where local changes in the velocities relax very quickly, as if the network were fixed. ${ }^{3}$ These results indicate that natural flocksat least the ones we are able to quantitatively observe-live in a regime where network rearrangements are slow, i.e., they are below the hydrodynamic regime. In this case, $\mathcal{H}$ gains the role of a pseudo-Hamiltonian, an effective representation of the forces and constraints acting on the degrees of freedom of the system, which effectively determines the probability distribution.

Hereafter we will explore possible mechanisms giving rise to speed and density waves even in the absence of network

\footnotetext{
${ }^{2}$ Nevertheless, there is evidence that in some cases it is still possible to have a statistical physics approach [62-64].

${ }^{3}$ This is the reason why inference methods based on static probability distributions give equivalent results to a full dynamical inference [65].
}

rearrangements. ${ }^{4}$ We will proceed in the following way: we will start from the pseudo-Hamiltonian Eq. (4) and modify it to allow for fluctuating speeds. Then we will associate to this Hamiltonian a dynamical equation for the velocities.

\section{Speed as the fundamental degree of freedom}

Speed fluctuations are usually neglected, and the modulus of the velocity is assumed equal to some $v_{0}$, the average speed of the flock, which is fixed by the birds' physiology. To study fluctuations around this average, the hard constraint must be abandoned, and an anchoring term must be added to the pseudo-Hamiltonian, as done in Refs. [43] and [44]. The pseudo-Hamiltonian describing the system then becomes

$$
\mathcal{H}=\frac{J}{4 v_{0}^{2}} \sum_{i j} n_{i j}\left|\mathbf{v}_{i}-\mathbf{v}_{j}\right|^{2}+\frac{g}{2 v_{0}^{2}} \sum_{i}\left(v_{i}-v_{0}\right)^{2},
$$

where $v_{i}=\left|\mathbf{v}_{i}\right|$. The first term describes the tendency of the individual velocities to adjust both direction and modulus to their neighbors, while the second forces the speed towards the mean physiological value $v_{0}$, introducing a speed control constant $g$.

In the highly polarized flocking phase, all the individuals move approximately in the same direction $\mathbf{n}$. For natural flocks, for example, the polarization $\Phi=1 / N\left|\sum_{i} \mathbf{v}_{i} /\right| v_{i} \mid$ is very large (of order 0.9 ) and the relative fluctuations both in flight directions and in speeds are very small [43]. We can write $\mathbf{v}_{i}=v_{i} \mathbf{s}_{i}$ (with $\left|\mathbf{s}_{i}\right|=1$ ) and express both the flight direction and the speed in terms of the fluctuations: $\mathbf{s}_{i}=$ $s_{i}^{L} \mathbf{n}+\pi_{i}$ and $v_{i}=v_{0}+u_{i}$. Assuming that the fluctuations are small we can expand in the $\boldsymbol{\pi}_{i}$ and $u_{i}$; one can easily see that to leading order the pseudo-Hamiltonian (5) splits into two terms:

$$
\begin{gathered}
\mathcal{H}=\mathcal{H}_{\mathrm{or}}\left(\left\{\boldsymbol{\pi}_{i}\right\}\right)+\mathcal{H}_{\mathrm{sp}}\left(\left\{u_{i}\right\}\right), \\
\mathcal{H}_{\mathrm{or}}=\frac{J}{2 v_{0}^{2}} \sum_{i j} n_{i j}\left|\boldsymbol{\pi}_{i}-\boldsymbol{\pi}_{j}\right|^{2}, \\
\mathcal{H}_{\mathrm{sp}}=\frac{J}{2 v_{0}^{2}} \sum_{i j} n_{i j}\left(u_{i}-u_{j}\right)^{2}+\frac{g}{2 v_{0}^{2}} \sum_{i} u_{i}^{2},
\end{gathered}
$$

one involving only orientations and the another involving only speed fluctuations. This decoupling allows us to concentrate on the speed part only. From now on, we will therefore forget about the flight directions and focus on the speed fluctuations (see Ref. [29] for a description of the orientational dynamics).

We notice that $\mathcal{H}_{\mathrm{sp}}$ is formally analogous to the Hamiltonian of a chain of harmonic oscillators in which every element, in addition to being connected to its nearest neighbors with strength $J$, has an additional spring that binds it to a fixed position (Fig. 1). Of course, in this formal analogy, the degree of freedom $u_{i}$ is a displacement with respect to a certain reference position, while in our case $u_{i}$ is a displacement

\footnotetext{
${ }^{4}$ Above some crossover scale (i.e., for very large flocks) the system would eventually enter the hydrodynamic regime. This crossover, which depends on the microscopic parameters of the system, has been investigated in Ref. [66].
} 


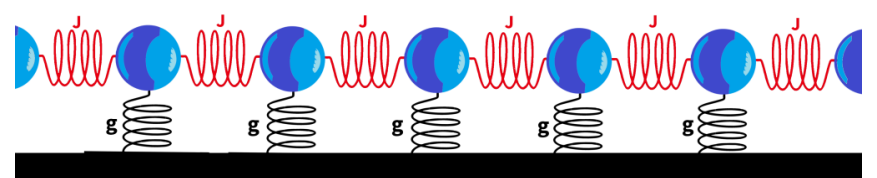

FIG. 1. Sketch of a chain of oscillator. Each oscillator, besides being connected to its first neighbors with strength $J$ (red spring), is also tied to a base with strength $g$ (black spring), which forces it to have a determined position.

(or, more properly, a fluctuation) with respect to a certain reference speed (typically, the physiological speed of the individual).

Assuming that the speed from bird to bird is smooth, we can take the continuous limit of this expression, in which the speed is a continuous function of the position in the flock, $\mathbf{x}$, and the time, $t$. First, we rewrite the expression for the speed Hamiltonian as

$$
\mathcal{H}_{\mathrm{sp}}=\frac{1}{2 v_{0}^{2}} \sum_{i, j=1}^{N}\left(J \Lambda_{i j}+g \delta_{i j}\right) u_{i} u_{j}
$$

where the matrix $\Lambda_{i j}$ has the form

$$
\Lambda_{i j}=-n_{i j}+\delta_{i j} \sum_{k=1}^{N} n_{i k}
$$

and represents the discrete version of the (negative) Laplacian operator. To see this let us suppose that the birds are in a line, and the relevant neighborhood is just the two nearest neighbors along the line. Then by labeling the birds by $n$ and each bird's neighbor by $n+1$ we can rearrange the terms in the sum (9) to give

$$
\mathcal{H}_{\text {sp }}=\frac{1}{2 v_{0}^{2}} \sum_{n=1}^{N} J\left(u_{n}-u_{n+1}\right)^{2}+g u_{n}^{2} .
$$

If the speed varies very slowly, we can picture it as a function of the position $x$ in the flock, $u(x)$, despite the fact that the birds are located at discrete position $x_{n}=n a$ where $a$ is the typical distance between the nearest birds. Then we have

$$
\mathcal{H}_{\mathrm{sp}} \simeq \frac{1}{2 v_{0}^{2}} \sum_{n=1}^{N} J a^{2}\left[\frac{\partial u(x)}{\partial x}\right]^{2}+g u(x)^{2} .
$$

Since the variations are smooth, we can turn the sum into an integral

$$
\mathcal{H}_{\mathrm{sp}}=\frac{1}{2 v_{0}^{2}} \int \frac{d x}{a} J a^{2}\left[\frac{\partial u(x)}{\partial x}\right]^{2}+g u(x)^{2} .
$$

By doing the same calculation with birds in a regular lattice in three-dimensional space rather then along a line, we can write $u(\mathbf{x}, t)=v(\mathbf{x}, t)-v_{0}$, and the Hamiltonian (8) finally takes the form

$$
\mathcal{H}_{\mathrm{sp}}=\int \frac{d^{3} x}{a^{3}}\left\{\frac{J a^{2} n_{c}}{2 v_{0}^{2}}[\nabla u(\mathbf{x}, t)]^{2}+\frac{g}{2 v_{0}^{2}} u^{2}(\mathbf{x}, t)\right\},
$$

where $n_{c}$ is the typical number of first neighbors with which each particle $i$ directly interacts.
The anchoring or control constant $g$ plays a fundamental role in determining the speed correlations. To see this we note that the pseudo-Hamiltonian (14) is Gaussian in the $u(\mathbf{x})$. One can then easily compute the statistical equilibrium averages and get [67]

$$
\left\langle u(\mathbf{x}) u\left(\mathbf{x}^{\prime}\right)\right\rangle \propto e^{-\left|\mathbf{x}-\mathbf{x}^{\prime}\right| / \xi_{\mathrm{sp}}},
$$

where the correlation length of speed fluctuations is given by

$$
\xi_{\text {sp }} \sim a \sqrt{J n_{c} / g} .
$$

In particular, $\xi_{\text {sp }}$ becomes infinite (and the correlation scalefree) at the critical point $g=0$. However, $g$ cannot be exactly zero, otherwise nothing fixes the mean speed of the birds. Nevertheless for small enough values of $g$ the system is effectively critical due to the finite size effects $\left(\xi_{\text {sp }} \propto L\right)$ [43].

The Langevin dynamics for speed fluctuations following from the pseudo-Hamiltonian just defined is

$$
\begin{aligned}
\frac{\partial u(\mathbf{x}, t)}{\partial t} & =-\frac{\delta \mathcal{H}_{\mathrm{sp}}}{\delta u(\mathbf{x}, t)}+\zeta(\mathbf{x}, t) \\
& =\frac{J a^{2} n_{c}}{v_{0}^{2}} \nabla^{2} u(\mathbf{x}, t)-\frac{g}{v_{0}^{2}} u(\mathbf{x}, t)+\zeta(\mathbf{x}, t),
\end{aligned}
$$

where $\zeta(\mathbf{x}, t)$ is a random white noise $\left\langle\zeta(\mathbf{x}, t) \zeta\left(\mathbf{x}^{\prime}, t\right)\right\rangle=$ $2 \eta T a^{3} \delta\left(\mathbf{x}-\mathbf{x}^{\prime}\right) \delta\left(t-t^{\prime}\right)$. The structure of this equation of motion is very different from what we would expect from a propagating phenomenon. Since $u$ is the fundamental degree of freedom, this equation is an overdamped first-order equation of the parabolic type $[48,68]$. This means that information travels sublinearly, $x \sim \sqrt{D t}$, and that a speed of propagation cannot even be defined. The diffusive structure of this equation is therefore unsuitable to describe the propagating phenomenon we expect.

\section{Hamiltonian dynamics and speed waves}

To obtain a theory able to describe propagating speed waves, we switch to an underdamped Hamiltonian dynamics. An Hamiltonian dynamics has the advantage of automatically implementing the symmetries present in the system and proves to be the key ingredient to reproduce propagation waves in the orientational degrees of freedom [29]. To this aim, we introduce a canonical pair of coordinates $(u, w)$, where $u$ are the speed fluctuations and $w$ is the generator of the transformation parametrized by $u$. This transformation corresponds to a translation in the speed, and it is the fundamental mechanism generating speed waves. Once we introduce the conjugated momentum $w$, we can build the full Hamiltonian for $u$ and $w$ by adding to the interaction term containing the speeds $\left(\mathcal{H}_{\mathrm{sp}}\right)$, a generalized kinetic term,

$\mathcal{H}=\int \frac{d^{3} x}{a^{3}}\left\{\frac{J a^{2} n_{c}}{2 v_{0}^{2}}[\nabla u(\mathbf{x}, t)]^{2}+\frac{g}{2 v_{0}} u^{2}(\mathbf{x}, t)+\frac{w^{2}(\mathbf{x}, t)}{2 \mu}\right\}$,

where $\mu$ is the inertia associated to the canonical pair $(u, w)$. It is important to note that $\mu$ is not the standard mass, but a generalized inertia that embodies the resistance of the bird to a change of $\dot{u}$. The symmetry generated by $w$ and parametrized 
by $u$ is a translation in the space of speed, which we may call a boost. The term $g u^{2}$ breaks this symmetry, thus giving to each individual a preferred speed, its physiological value. The interesting point is that, when a system is highly polarized, the boost transformation we are talking about becomes conceptually quite close to a Galilean transformation (clearly, this is far from true if the polarization is low). Since flocks are highly polarized, this analogy is fair, and in this context we see then that the $g u^{2}$ term breaks Galilean invariance. This has the rather interesting consequence that the scale-free point $g=0$ identifies with the point which restores Galilean invariance in the system.

Having an inertial term allows us to consider a dynamics, given by the canonical equations of motion:

$$
\begin{aligned}
\frac{\partial u(\mathbf{x}, t)}{\partial t} & =\frac{\delta \mathcal{H}}{\delta w(\mathbf{x}, t)}, \\
\frac{\partial w(\mathbf{x}, t)}{\partial t} & =-\frac{\delta \mathcal{H}}{\delta u(\mathbf{x}, t)}-\eta \frac{\partial u(\mathbf{x}, t)}{\partial t}+\zeta(\mathbf{x}, t),
\end{aligned}
$$

where we have reinstated a friction term $\eta$ and noise, to get a set of equations containing both conservative and dissipative terms. From this pair of equations follows the equation of motion for the speed,

$$
\begin{aligned}
& \frac{\partial^{2} u(\mathbf{x}, t)}{\partial t^{2}}+2 \gamma \frac{\partial u(\mathbf{x}, t)}{\partial t}+\omega_{0}^{2} u(\mathbf{x}, t)-c^{2} \nabla^{2} u(\mathbf{x}, t) \\
& \quad=\zeta(\mathbf{x}, t)
\end{aligned}
$$

where $c^{2}=J a^{2} n_{c} / \mu$ is the phase velocity of the propagating waves, $\gamma=\eta / 2 \mu$ is the reduced friction. and $\omega_{0}^{2}=g / \mu$ is the natural frequency (i.e., the frequency with which the system would oscillate in the absence of the social force). This is a second-order equation of the hyperbolic type, suitable to represent propagating phenomena [68] and known in the literature as the telegraph equation $[47,48]$. It can be further simplified by introducing a new field $v(\mathbf{x}, t): u(\mathbf{x}, t)=$ $e^{-\gamma t} v(\mathbf{x}, t)$ so that the terms containing $\partial v / \partial t$ drop out in the equation for $v$. Then for the homogeneous case we get

$$
\frac{\partial^{2} v(\mathbf{x}, t)}{\partial t^{2}}=c^{2} \nabla^{2} v(\mathbf{x}, t)+\varepsilon^{2} v(\mathbf{x}, t)
$$

where

$$
\varepsilon^{2}=\gamma^{2}-\omega_{0}^{2}
$$

Note that $\varepsilon^{2}$ can be positive or negative depending on whether the friction dominates over speed control or vice versa; the use of the square notation derives from the definition given in the standard telegraphic equation, where $\varepsilon^{2}$ is defined semipositive. In this form it is clear that $\varepsilon^{2}=0$ is a critical value: if the parameters are such that $\varepsilon^{2}=\eta^{2} / 4 \mu^{2}-g / \mu=$ 0 , Eq. (21) reduces to the classical wave equation. This case in which the physical constants can be adjusted to eliminate the dispersion corresponds in literature to the lossless transmission line case [49]. Besides reducing the distortion of the signal, this point has the interesting property of minimizing the time required for the system to return to the unperturbed state. It thus represents an optimal situation for the information transfer. From a biological point of view it would be an extremely useful mechanism. It is therefore important to examine in depth the working principle and properties of this mechanism.

\section{THE DISPERSION RELATION}

In order to better understand the differences between the Langevin dynamics [Eq. (17)] and the Hamilton dynamics [Eqs. (19)] and their role in the complete dynamical equation (20), it is useful to study the dispersion relation (see Fig. 2).

\section{A. Langevin versus Hamilton dynamics}

For Langevin dynamics the dynamic equation is of first order in time, and accordingly the frequency is purely imagi-
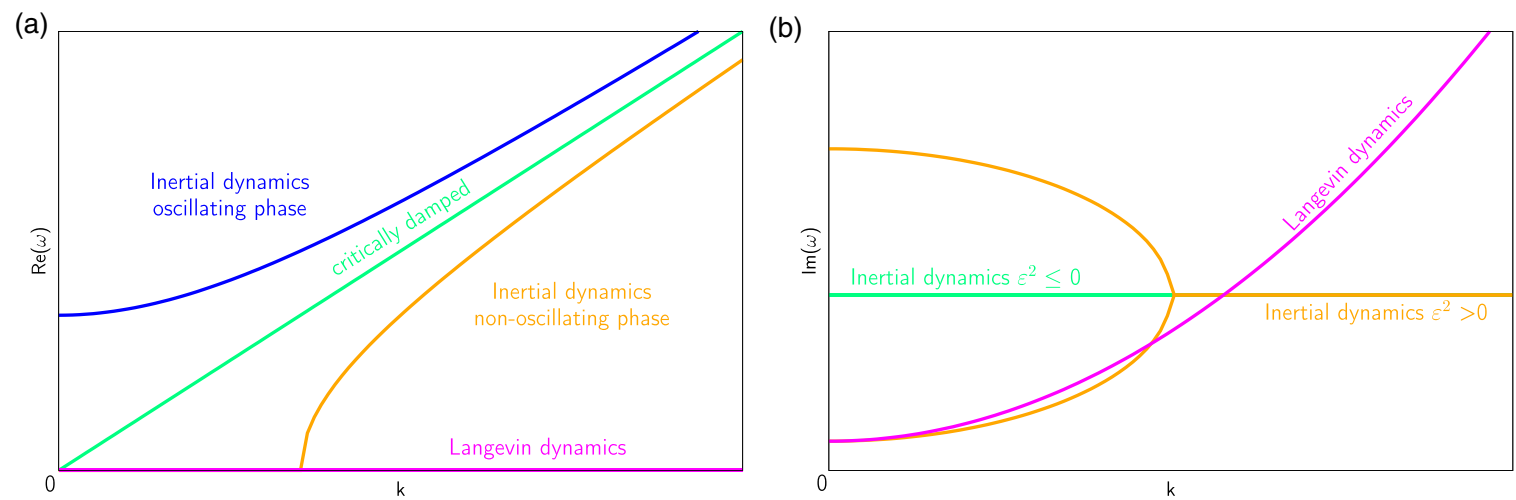

FIG. 2. Sketch of the dispersion law. (a) It is drawn the real part of the frequency $\omega$ for different cases. If $\varepsilon^{2}<0$ (blue line), we are in the oscillating phase and there is propagation for every value of $k$; if $\varepsilon^{2}>0$ (orange line), the system is nonoscillating and there is propagation only for $k>k_{0}=\frac{\varepsilon}{c}$; if $\varepsilon^{2}=0$ (green line), we are at the critical damping and there is always linear propagation; for Langevin dynamics (fuchsia line) the real part of the frequency is zero. (b) The imaginary part of the frequency $\omega$ for different cases. The green line represents the oscillating and critically damped situations in which $\operatorname{Im}(\omega)$ is constant; the orange line represents the nonoscillating regime in which the $\operatorname{Im}(\omega)$ is constant only for $k>k_{0}$ and grows quadratically with $k$ for small values of $k$. 
nary:

$$
\omega=i\left(D k^{2}+\omega_{0}\right)
$$

where

$$
D=\left(J a^{2} n_{c}\right) /\left(v_{0}^{2} \eta\right), \quad \omega_{0}=g /\left(v_{0} \eta\right) .
$$

The vanishing of the real part corresponds to the fact that there is no propagation but only exponential damping. The (imaginary) frequency has a gap $\omega_{0}$ plus a quadratic diffusive term $D k^{2}$. As a consequence all the modes are overdamped, and a disturbance spreads diffusively through the system.

Introducing the conjugate momentum of the speed and the generalized inertia gives, as we saw, an equation of second order in time and space [Eq. (21)]. The dispersion polynomial associated to it is of second order in both frequency and momentum,

$$
\omega=i \gamma \pm \sqrt{c^{2} k^{2}-\varepsilon^{2}} .
$$

From this dispersion law it is clear that the parameter $\varepsilon^{2}$ plays a fundamental role in determining the type of propagation. If $\varepsilon^{2}<0$ the argument of the square root is always positive, and $\omega$ has a real part even for $k=0, \operatorname{Re} \omega(k=0)= \pm|\varepsilon|$. This is the oscillating zone: there is propagation for every $k$, and the dispersion relation is quadratic for small $k$, approaching a linear behavior at large $k$. On the other hand, if $\varepsilon^{2}>0$, the argument of the square root changes sign with $k$. In this case the system is nonoscillating, and there is propagation only for $k>k_{0}=\varepsilon / c$. However, for large values of $k$ we recover again a linear dispersion law.

It is crucial to note that the particular value $\varepsilon^{2}=0$ guarantees linear propagation at all values of $k, \operatorname{Re} \omega= \pm c k$, but with some damping $\gamma$, independent of $k$. In this case the real parts of the two roots coincide since the damping factor $\gamma$ and the natural frequency $\omega_{0}$ of the system perfectly balance. We have already noticed that the speed Hamiltonian is analogous to the one of a chain of oscillators. The picture we have just described, for $\varepsilon^{2}=0$, has an interesting connection with what happens even in a single damped harmonic oscillator, and in particular with the definition of critical damping. It is useful to explore the meaning of this toy case in order to simply catch the fundamental properties of this particular value.

\section{B. Toy model: Critical damping and minimum return time}

The damped harmonic oscillator (DHO) represents well many different physical situations (mechanical oscillator, RLC circuit, etc.). We would like to use it as a paradigmatic situation for what happens in the speed waves model we derived. The well-known equation of motion can be written as [69]

$$
\ddot{x}(t)+2 \gamma \dot{x}(t)+\omega_{0}^{2} x(t)=0,
$$

where we have introduced the damping constant $\gamma=\eta / 2 m$ and the natural frequency $\omega_{0}=\sqrt{k / m}$, while $m$ is the inertia, $\eta$ the viscosity, and $k$ the elastic constant or stiffness. The dispersion polynomial reads

$$
\omega=i \gamma \pm \sqrt{\omega_{0}^{2}-\gamma^{2}}=i \gamma \pm \sqrt{-\varepsilon^{2}}
$$

The shape of the solution depends crucially on the value of $\varepsilon^{2}$, that is, on the balance between reduced viscosity $\gamma$ and natural frequency $\omega_{0}$. There are two solutions separated by a critical point. For $\varepsilon^{2}<0$ we are in the underdamped regime, meaning that inertia (and stiffness) dominate over viscosity; here the solution displays a clear oscillatory behavior. For $\varepsilon^{2}>0$ the DHO enters in the overdamped regime, where the two roots are purely imaginary. In this regime viscosity dominates, and the solution does not show oscillations but falls to zero exponentially. At precisely $\gamma=\omega_{0}$, namely, $\varepsilon^{2}=0$, one has critical damping, which represents the boundary between underdamping and overdamping. As in the overdamped case, the solution shows no oscillations, but for this particular condition the system minimizes the return time $\tau$ to the rest position. A critically damped system therefore relaxes a perturbation as fast as possible, without oscillating [69].

There are many situations in which one wants passing disturbances to end as quickly as possible (shock absorbers of a car, closing system of a door, etc.). In all these cases it is necessary to adjust the parameters so that the damping is as close as possible to critical. Regarding collective response, it could be very important that the reaction to external perturbations is performed in the shortest possible time, spending as little energy as possible. In particular, such an optimization seems sensible in the case of a flock in motion: it would appear reasonable to avoid situations where, after responding to a perturbation, a particular bird would start oscillating around the cruising speed, or take a very long time to return to that value. A critical damping on the propagation of speed fluctuations would ensure a cohesive and efficient movement. However, our full equation for the speed is more complicated than this simple case because we have a field (infinite degrees of freedom), rather than one degree of freedom; this is why the extra term $k^{2}$ arises in the full dispersion relation (25). Next we will investigate if this intuition is supported by the solution of the speed waves model.

\section{EXACT SOLUTION OF THE SPEED WAVE EQUATION IN $d=1$}

We now study the full solution of the speed waves model. We start from the simplest case, that is, from the solution in dimension $d=1$. Again for reasons of simplicity we will assume that the system is infinite. Although this may seem an unrealistic approximation, it has no consequences for the purpose of the study, since our interest here is the way the signal propagates through space, and this propagation does not rely on the infinite nature of the system. In particular, we would like to understand if and how a critical damping regime is reflected by the mathematics of the problem.

\section{A. Wave and wake}

The solution for general initial conditions,

$$
\left.u(x, t)\right|_{t=0}=\phi(x),\left.\quad \frac{\partial u(x, t)}{\partial t}\right|_{t=0}=\psi(x),
$$


is given by $[47,48]$

$$
\begin{aligned}
u(x, t)= & e^{-\gamma t}\left\{\frac{\phi(x-c t)+\phi(x+c t)}{2}+\frac{1}{c} \int_{x-c t}^{x+c t} d x^{\prime}\left[\gamma \phi\left(x^{\prime}\right)+\psi\left(x^{\prime}\right)\right] \mathrm{I}_{0}\left[\frac{\varepsilon}{c} \sqrt{c^{2} t^{2}-\left(x^{\prime}-x\right)^{2}}\right]\right. \\
& \left.-\frac{\varepsilon t}{2} \int_{x-c t}^{x+c t} d x^{\prime} \frac{\phi\left(x^{\prime}\right) \mathrm{I}_{1}\left[\frac{\varepsilon}{c} \sqrt{c^{2} t^{2}-\left(x^{\prime}-x\right)^{2}}\right]}{\sqrt{c^{2} t^{2}-\left(x^{\prime}-x\right)^{2}}}\right\}
\end{aligned}
$$

where $I_{0}$ and $I_{1}$ are modified Bessel function of the first kind. Since we are interested in the way a localized perturbation propagates, we consider the initial conditions with a pulse at $x=0$ :

$$
\left.u(x, t)\right|_{t=0}=u_{0} \delta(x),\left.\quad \frac{\partial u(x, t)}{\partial t}\right|_{t=0}=0 .
$$

In this case we can write the solution as

$$
u(x, t)=u_{0} e^{-\gamma t}\left\{\frac{\delta(x-c t)+\delta(x+c t)}{2}+\left[\frac{\gamma}{c} \mathrm{I}_{0}\left(\frac{\varepsilon}{c} \sqrt{c^{2} t^{2}-x^{2}}\right)-\frac{\varepsilon t}{2} \frac{\mathrm{I}_{1}\left(\frac{\varepsilon}{c} \sqrt{c^{2} t^{2}-x^{2}}\right)}{\sqrt{c^{2} t^{2}-x^{2}}}\right] \theta(c t-|x|)\right\} .
$$

For $\varepsilon^{2}<0$ the modified Bessel functions can be replaced by Bessel functions of the first kind $\mathrm{J}_{0}$ and $\mathrm{J}_{1}$. Finally, for $\varepsilon^{2}=0$ the solution reduces to

$$
u(x, t)=u_{0} e^{-\gamma t}\left\{\frac{\delta(x-c t)+\delta(x+c t)}{2}+\frac{\gamma}{c} \theta(c t-|x|)\right\}
$$

The main effect of viscosity is the presence of the overall damping factor $e^{-\gamma t}$. Looking at the terms within braces, we see that the first two terms represent pulses propagating left and right with speed $c$; this term would be present also without anchoring (standard wave equation). The remaining term instead introduces another phenomenon: the wave leaves a wake. Even after the wave front has passed, an effect that originates from all the points where the initial condition is different from zero is present at all points within a distance $t / c$ from them. This wake vanishes exponentially in time $[47,48]$. As a consequence a given point does not return instantaneously to its equilibrium position (as it would in a d'Alembert wave), but there is a tail in time, whose structure depends on the value of the parameters (see Fig. 3).

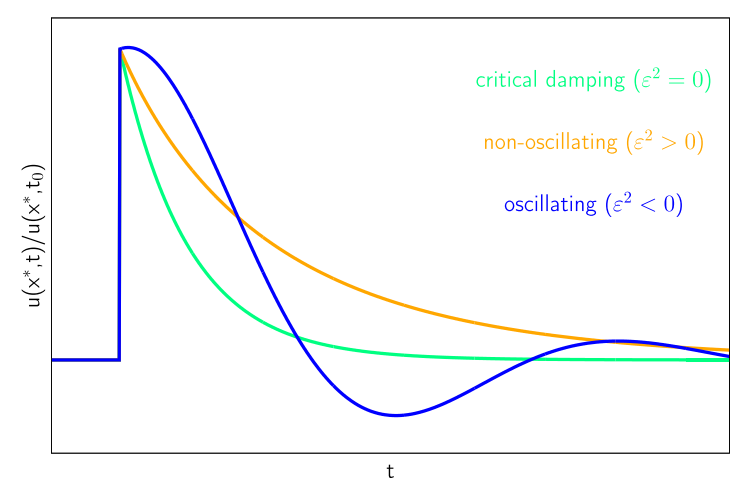

FIG. 3. Different solutions of the speed equation in $d=1$. Depending on the value of $\varepsilon^{2}$ the solution will go to zero differently: at $\varepsilon^{2}=0$ (green line) it reaches zero in the fastest way without oscillating; in the nonoscillating regime (orange line), $\varepsilon^{2}>0$ the solution goes to zero more slowly, while in the oscillating case (blue line) the field displays oscillations before going to the original value.

\section{B. Return time}

To understand how the field returns to its unperturbed value, it is necessary to define the return time $\tau$. Up to this point we have not considered the effect of noise. We can suppose that, in the limit of small noise, the shape of the full solution will not differ much from the homogeneous one (31). On the other hand, it is legitimate to assume that the unperturbed state will be different from that of the homogeneous case where it is equal to zero, but that the solution will decay to a certain averaged value due to noise fluctuations. Hence we will define $\tau$ as the time it takes for the solution to decay to a certain level $1 / q$ after the arrival of the wavefront. Since the signal arrives at $x^{\star}$ at a position-dependent time $t_{0}=x^{\star} / c$, our definition for the return time at $x=x^{\star}$ reads

$$
u\left(x^{\star}, t_{0}\left(x^{\star}\right)+\tau\right)=1 / q .
$$

This definition is appropriate for the nonoscillating phase, but when $\varepsilon^{2}<0$, the solution oscillates and has an infinity of zeros, and the above definition would not give a unique value of $\tau$; on the other hand, taking the smallest solution is not appropriate because it will be dominated by the period of the oscillations (at high frequency at least). Therefore in the oscillating case we simply disregard oscillations and assume that the return time is determined by the exponential decay envelope. Hence for $\varepsilon^{2}<0$ we ignore the oscillating Bessel functions and find

$$
\tau=-t_{0}+\frac{1}{\gamma} \log \left(\frac{u_{0} q \gamma}{c}\right) .
$$

This solution is also good for the critical line $\varepsilon^{2}=0$ as can be seen from Eq. (32).

Along the critical line, the return time $\tau$ is characterized by a first region where it is zero, followed by a zone in which it grows up to a maximum at $\gamma=c e / u_{0} q$ and then decreases until it vanishes again. However, the two regions of vanishing 
(a)
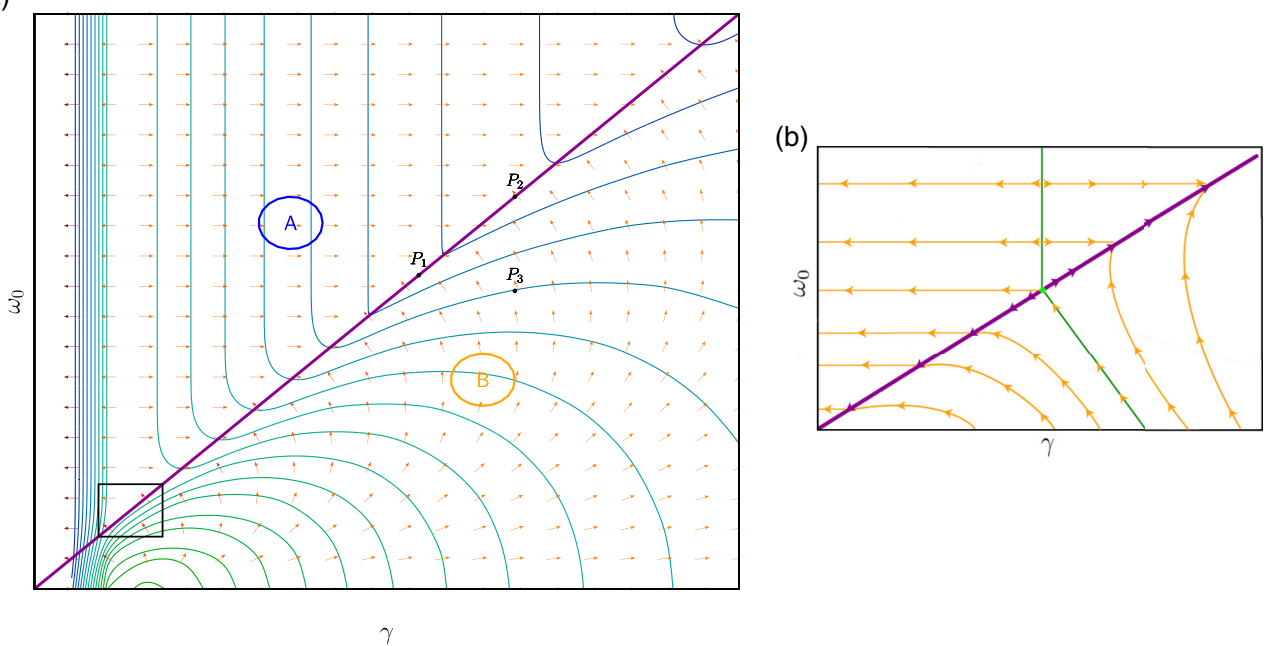

FIG. 4. (a) Level lines of $\tau\left(\gamma, \omega_{0}\right)$ are drawn with colors from blue for lower values to green for the higher ones. The vector field is given by the negative gradient of the function; here as well the color is the intensity of the field, ranging from yellow for lower values to dark red for the highest ones. The violet line represents the critical line. (b) Zoom of the gradient flow near the saddle point dividing the transparent zone from the intermediate zone [the region inside the black rectangle of panel (a)]. The green line represents the separatrix, which divides the steepest descent dynamics.

$\tau$ are qualitatively different. The first region, which we call the transparent zone, is characterized by very small values of $\gamma$. Since the height of the wake is proportional to $\gamma$, when this is small all the wake falls below the noise fluctuation level, and the only relevant perturbation is the traveling $\delta(x \pm c t)$, which has $\tau=0$. The second region where the return time is zero is instead characterized by very large values of the damping $\gamma$ or large value of $t_{0}$. This we call the opaque zone: here $t_{0} \gg$ $1 / \gamma$, and therefore the signal is strongly damped and cannot reach the position $x^{\star}$, since the amplitude of all the terms is below the noise threshold. A return time is not even definable (we would have negative values of $\tau$ ); we conclude that in the transparent region, the propagation is effectively d'Alembert.

We do not have an analytic expression for $\tau$ for $\varepsilon^{2}>0$, but close to the critical line we can expand the Bessel functions for small values of the argument and solve Eq. (33) recursively to get

$$
\begin{aligned}
\tau= & \tau_{0}+\frac{\varepsilon^{2}}{2 \gamma^{2}}\left(\frac{t_{0}}{2}+\frac{\tau_{0}}{2}+\gamma t_{0} \tau_{0}+\gamma \frac{\tau_{0}^{2}}{2}\right) \\
= & t_{0}+\frac{1}{\gamma} \log \left(\frac{u_{0} q \gamma}{c}\right) \\
& +\frac{\varepsilon^{2}}{4 \gamma^{3}}\left\{\log \left(\frac{u_{0} q \gamma}{c}\right)\left[1+\log \left(\frac{u_{0} q \gamma}{c}\right)\right]-\gamma^{2} t_{0}^{2}\right\} .
\end{aligned}
$$

This expression shows that $\tau$ grows when $\varepsilon^{2}$ grows at fixed $\gamma$. Hence, $\varepsilon^{2}=0$ minimizes the return time at fixed $\gamma$. Let us clarify this point further.

\section{The critical line as an attractor of return time minimization}

To better understand the significance of the critical line, $\varepsilon^{2}=0$, we consider the contour lines of $\tau$ and the gradient field

$$
-\nabla \tau=\left(-\frac{\partial \tau}{\partial \gamma},-\frac{\partial \tau}{\partial \omega_{0}}\right)
$$

in the $\left(\gamma, \omega_{0}\right)$ plane [Fig. 4(a)]. The figure shows that the $\varepsilon^{2}=0$ line is an attractor for a gradient descent dynamics of $\tau$. Although there are points outside the critical line that have a return time lower than some points on it, the gradient flows toward the critical line, so that a dynamic that tries to minimize the return time with local moves will end up along the line $\varepsilon^{2}=0$. For example, the point $P_{3}$ in Fig. 4(a) has a return time lower than $P_{1}$. However, the gradient flow does not take $P_{1}$ towards $P_{3}$; it rather takes both toward the point $P_{2}$. To see this, consider the plane $\left(\gamma, \omega_{0}\right)$ and call (A) the oscillating and (B) the nonoscillating regions [see Fig. 4(a)]. For the critical line to be an attractor, the gradient lines in its neighborhood must point towards it. Therefore one must have

$$
\begin{aligned}
& -\left.\frac{\partial \tau_{\mathrm{A}}}{\partial \gamma}\right|_{\varepsilon^{2}=0}>-\left.\frac{\partial \tau_{\mathrm{A}}}{\partial \omega_{0}}\right|_{\varepsilon^{2}=0}, \\
& -\left.\frac{\partial \tau_{\mathrm{B}}}{\partial \omega_{0}}\right|_{\varepsilon^{2}=0}>-\left.\frac{\partial \tau_{\mathrm{B}}}{\partial \gamma}\right|_{\varepsilon^{2}=0} .
\end{aligned}
$$

In region $\mathrm{A} \tau$ is given by (34), therefore

$$
-\left.\frac{\partial \tau_{\mathrm{A}}}{\partial \gamma}\right|_{\varepsilon^{2}=0}=\frac{1}{\gamma^{2}}[\log (\alpha \gamma)-1]>0=-\left.\frac{\partial \tau_{\mathrm{A}}}{\partial \omega_{0}}\right|_{\varepsilon^{2}=0},
$$

where $\alpha=u_{0} q / c$. This condition is verified for $\gamma>e / \alpha$, i.e., outside the transparent zone. In region $B$ the return time is given by (35), so that the gradient is

$$
\begin{aligned}
& -\left.\frac{\partial \tau_{\mathrm{B}}}{\partial \omega_{0}}\right|_{\varepsilon^{2}=0}=\frac{1}{2 \gamma^{2}}\left[\log (\alpha \gamma)+\log ^{2}\left(\frac{u_{0} q \gamma}{c}\right)-\gamma^{2} t_{0}^{2}\right], \\
& -\left.\frac{\partial \tau_{\mathrm{B}}}{\partial \gamma}\right|_{\varepsilon^{2}=0}=\frac{1}{2 \gamma^{2}}\left[\log (\alpha \gamma)-\log ^{2}(\alpha \gamma)+\gamma^{2} t_{0}^{2}-2\right],
\end{aligned}
$$


yielding

$$
\log ^{2}(\alpha \gamma)+1>\gamma^{2} t_{0}^{2},
$$

which is certainly verified if $\tau_{0}>0$, that is for $\log (\alpha \gamma)>$ $\gamma t_{0}$. This means that every point close to the part of the critical line with a positive return time will flow to the critical line. We conclude that the critical line is an attractor for the gradient dynamics of $\tau$. We may metaphorically view the (rather complicated) function $\tau\left(\gamma, \omega_{0}\right)$ as the main rib of a leaf, which is an attractor for a water drop [Fig. 4(c)], although the situation here is a bit more complicated because of the nontrivial critical line structure.

The maximum of $\tau$ on the critical line is a very special saddle point, because the flow field is not analytic at it: there is a separatrix that divides the basins of attraction of the transparent and of the opaque zone [Fig. 4(b)]. So, depending on whether one starts to the left or to the right of the separatrix, the gradient flow will drive one to the transparent zone or in the opaque zone of the critical line, respectively. Clearly in a biological system the parameters are fixed and there is no gradient dynamics of $\tau$. However, if we admit that the condition of critical damping is an advantage for biological systems, we could speculate that in the course of evolution the parameters describing these systems are being pushed towards the critical damping line. We expect that a real system, and in particular the one we want to describe, lies close to the transparent zone: here the signal passes with weak attenuation and arrives still strong in every part of the system.

\section{HOW TO LOOK FOR EVIDENCE OF SPEED WAVES IN EXPERIMENTAL DATA?}

To analyze how information propagates in a biological system directly, one has to observe an actual disturbance propagating in space and time. However, naturally occurring disturbances may be relatively rare, and it is not always fea- sible to generate and artificial disturbance. Another, indirect, way, is to analyze the spontaneous fluctuations of the system, that is, to study dynamical correlations. Indeed, qualitative features of the structure of the dynamical equations should leave identifiable traces in the shape of time correlations. We have seen that the dynamic equations are quite different depending on the presence or absence of inertial terms; let us show how this is reflected in the dynamic correlation function.

\section{A. Spatiotemporal correlations}

We will focus on the intermediate scattering function, which is quite easy to compute at the experimental level $[70,71]$,

$$
\begin{aligned}
C(k, t) & =\int d \mathbf{x} e^{-i \mathbf{k} \cdot \mathbf{x}} C(r, t) \\
& =\int d \mathbf{x} e^{-i \mathbf{k} \cdot \mathbf{x}}\left\langle u\left(\mathbf{x}, t_{0}\right) u\left(\mathbf{x}+r, t_{0}+t\right)\right\rangle,
\end{aligned}
$$

The spatiotemporal correlation function is a very useful tool, because its properties are entirely determined by the dispersion relation, which in turn mirrors the structure of the dynamical equation [70]. Hence, one can infer from the behavior of $C(k, t)$ a lot of information about the dynamics of a system. For the technical mathematical steps connecting the correlation function to the dispersion relation we refer the reader to Ref. [70].

In the case of Langevin dynamics (no speed waves) [Eqs. (17) and (23)], $C(k, t)$ has the form [see Fig. 5(a)]

$$
C(k, t)=\frac{2 T}{D k^{2}+\omega_{0}} e^{-\left(D k^{2}+\omega_{0}\right) t},
$$

where $T$ is the generalized temperature, and $D$ and $\omega_{0}$ have been defined in (24). One can easily read the Langevin dispersion relation (23) from the form of the correlation.
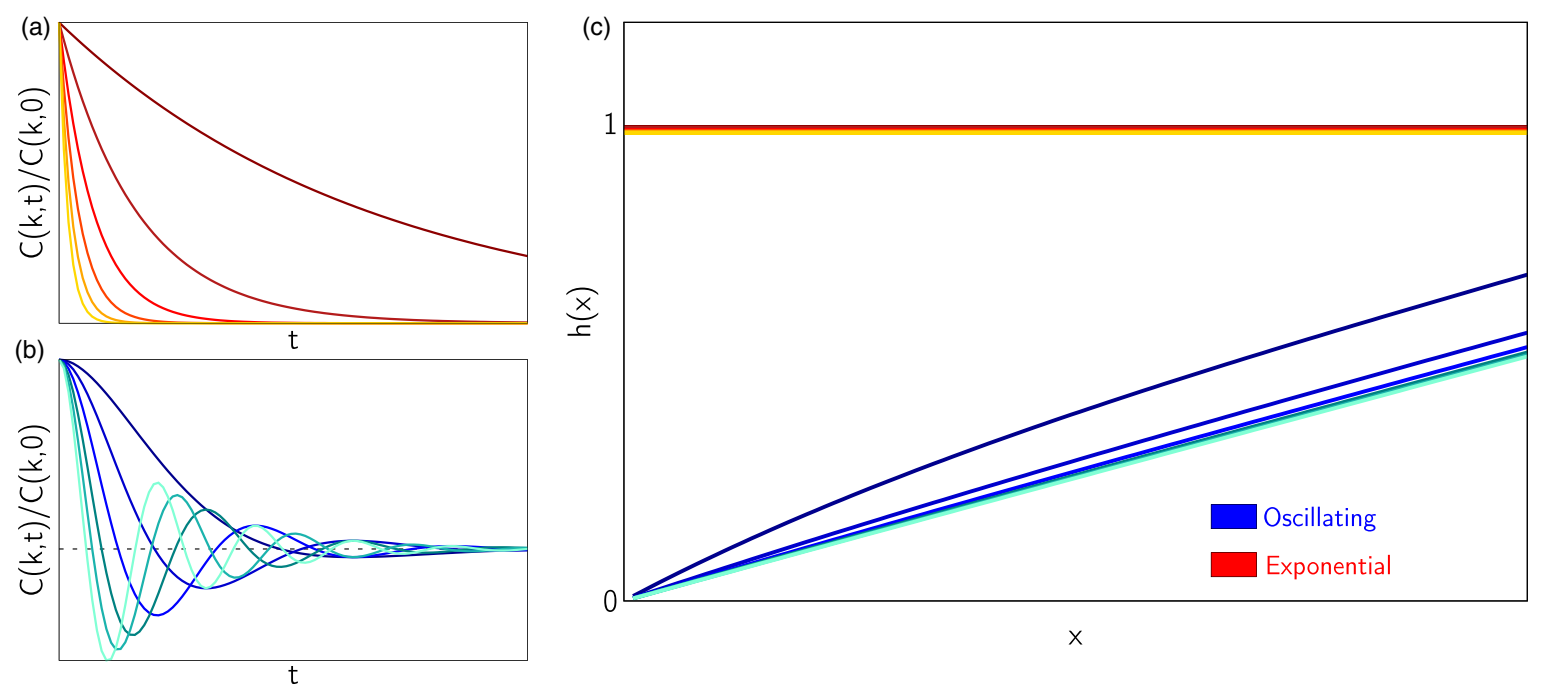

FIG. 5. (a) $C_{k}(t)$ in the nonoscillating regime $\left(\varepsilon^{2}>0\right)$ of the inertial dynamics for $k<k_{0}$ and for every value of $k$ in the Langevin dynamics. The colors represent different values of $k$, ranging from dark red for small values of $k$, to yellow for high $k$. (b) $C_{k}(t)$ in the inertial case for every value of $k$ if $\varepsilon^{2} \geqslant 0$ and only for $k>k_{0}$ if $\varepsilon^{2}>0$. The colors represent different values of $k$, ranging from dark blue for small values of $k$ to aquamarine for high $k$. (c) To better quantify the difference between inertial and noninertial system we define the function $h(x)$ (see Ref. [71]), which has a different form depending on whether the function is exponential-like or has a vanishing first derivative. 
On the other hand, for the inertial dynamics of the speed wave equation $\left(\varepsilon^{2}<0\right)$ the correlation function is given by [see Fig. 5(b)]

$$
\begin{aligned}
C(k, t)= & \frac{\eta T}{\left(\frac{\tilde{f} k^{2}}{\mu}+\omega_{0}^{2}\right)} e^{-\gamma t} \\
& \times\left\{\frac{\sin \left(\sqrt{\frac{\tilde{f} k^{2}}{\mu}-\varepsilon^{2}} t\right)}{\sqrt{\frac{\tilde{\jmath} k^{2}}{\mu}-\varepsilon^{2}}}+\frac{\cos \left(\sqrt{\frac{\tilde{f} k^{2}}{\mu}-\varepsilon^{2}} t\right)}{\gamma}\right\},
\end{aligned}
$$

where $\tilde{J}=J a^{2} n_{c}$. In the overdamped regime $\left(k<k_{0}=\varepsilon / c\right.$, $\varepsilon^{2}>0$ ) the trigonometric functions must be replaced by the respective hyperbolic functions of argument $\left[\sqrt{\varepsilon^{2}-\left(\tilde{J} k^{2}\right) / \mu} t\right]$. The form of the correlation function is considerably simpler than that of the full solution of the equation [Eq. (31)]. The correlation function refers only to a specific $k$ mode, while in the total solution all the modes are added giving rise to the Bessel functions.

\section{B. The fingerprint of inertial dynamics}

We see that Langevin dynamics displays plain exponential relaxation, while inertial systems have a nonexponential oscillating correlation function. At first sight this may seem an obvious difference, very easy to detect from empirical data. However, the situation is more complex. First, empirical data typically derive from real three-dimensional trajectories, which normally are not available for long times (the flock gets out of the field of view of our apparatus); if we have the correlation only for medium-short times, it may be impossible to detect the oscillations, even if inertia (and therefore speed waves) are present. Second, if the system is close to critical damping, then there are no oscillations, even if speed waves are present! Hence, using oscillations as an empirical landmark of inertia and propagating waves is not a good idea.

On the other hand, there is a feature of the correlation that is visible also for short times and that depends exclusively on the order of the dispersion relation (first versus second order), namely, on the number of poles in the complex $\omega$ plane of the the correlation function. This feature is the first time derivative of the correlation for $t \rightarrow 0$ [71]. If the dispersion relation is of the first order, as in the Langevin case, then the derivative of the correlation in zero is finite, while if the dispersion relation is of the second order, as in the speed waves case, the derivative must go to zero. In order to quantitatively perform this analysis we can define the function,

$$
h(x)=-\frac{1}{x} \log \left[\frac{C(x)}{C(0)}\right], \quad x \equiv t / t_{k},
$$

where $t_{k}$ is the characteristic timescale of the correlation, and study it in the interval $x \in[0,1]$, that is, for times $t<t_{k}$. For purely exponential relaxation $h(x) \rightarrow 1$ for $x \rightarrow 0$, while a flat time correlation gives $h(x) \rightarrow 0$ in the same limit. Furthermore, this function prevents us from calculating the derivative of data at a particular point that can have a large error. Once we have computed this function for experimental data, if one has $h(x) \rightarrow 0$, then it is quite fair to say that the data have been generated by a dynamical equation that has inertial terms, and therefore they are in a good agreement with the speed wave model. An experimental effort towards collecting this kind of data is currently under way.

\section{WHAT KIND OF CRITICAL DAMPING?}

The concept of critical damping in the context of collective behavior was first introduced and studied in 2010 by Paley and coworkers [72], which we now compare with our approach.

The first and most crucial difference between the two studies is that Paley and coworkers propose a one-dimensional mathematical model directly for the position, rather than for the speed; hence, in Ref. [72] the mechanism of imitation, typical of collective behavior, amounts to imitating the position of the neighbors rather than their speed. This is clearly visible in the mathematical expression of the model that they proposed, which is a second-order dynamics for the positions,

$$
\ddot{q}_{i}=\sum_{j \in n_{i}}-J\left[q_{i}-q_{j}-(i-j) q_{0}\right]-2 \xi \sqrt{J}\left(\dot{q}_{i}-\dot{q}_{j}\right),
$$

where $q_{i}$ is the position of the individual $i, \dot{q}_{i}$ its velocity, $J$ is the spring constant, $|i-j| q_{0}$ the rest length, while $2 \xi \sqrt{J}$ is the damping coefficient and $\xi>0$ [the inertia in (45) is the normal mechanical mass, which is set to one]. Instead of an anchoring term breaking the translational symmetry (translation in the speed in our case), (45) has a linear damper connecting the particles. In order to make the comparison with our equation, we rewrite (45) in the continuous limit,

$$
\frac{\partial^{2} q(\mathbf{x}, t)}{\partial t^{2}}+2 \xi c \nabla^{2} \frac{\partial q(\mathbf{x}, t)}{\partial t}-c^{2} \nabla^{2} q(\mathbf{x}, t)=\zeta(\mathbf{x}, t),
$$

where $q(\mathbf{x}, t)$ is the displacement field of the particles, $c=$ $J a^{2} n_{c}$, and $\zeta$ is a noise. This equation must be compared to our Eq. (20). The dispersion relation associated to (46) has the solution

$$
\omega=i \xi c k^{2} \pm c k \sqrt{1-\xi^{2} k^{2}} .
$$

For $k<1 / \xi$ the frequency has a real part (propagating modes), while for $k>1 / \xi$ the equation is overdamped; these two regimes are separated by a critical damping value, $k=$ $1 / \xi$, and in Ref. [72] it is discussed how this edge is influenced by the connectivity of the network. Our dispersion relation (25) can be rewritten as

$$
\omega=i \gamma \pm c k \sqrt{1-\frac{\varepsilon^{2}}{c^{2} k^{2}}} .
$$

Here too there is a critical damping edge, $k=\varepsilon / c$, but its role is the opposite than in (47): the frequency is real for large $k$ and purely imaginary for low $k$.

To conclude, in the context of Ref. [72] critical damping does not concern the balance between inertia and dissipation, as in our study, but a transition between propagating and nonpropagating modes in $k$ space. This type of definition of critical damping is not what impacts the general solution of the dynamical equation in real space, which is found by summing over all $k$ modes: solutions (31) depend only on $\varepsilon$, that is, on the balance between inertia and dissipation, and the critical damping value corresponds to $\varepsilon=0$, of which there is no analog in the model developed in Ref. [72]. 


\section{CONCLUSIONS}

We proposed a model for characterizing the propagation of speed fluctuations within highly polarized biological systems (flocks). The resulting second-order dynamical equation involves inertia, dissipation, interaction strength, and a symmetry-breaking term anchoring each individual to its physiological speed value. In general this equation has both underdamped and overdamped modes, giving rise to a complex structure of the general solution. However, we found that along a certain line in the space of parameters, when dissipation and inertia balance, the return time to the unperturbed state after a signal has passed is minimized. This is the critical damping line. We solved the equation exactly in one dimension and showed that the critical damping line is an attractor for a steepest descent dynamics of the return time. Finally, we proposed a method with which to assess, through an analysis of the experimental data, the validity of this model: by studying the dynamic correlations for speed, it should be possible to verify the presence or absence of inertial terms in the dynamics and to refute or validate our model accordingly.

Critical damping is quite a compelling concept at the biological level, especially in the case of speed waves. Let us consider a flock traveling unperturbed at a certain cruising speed. At some point an individual at the back of the flock detects a perturbation (as a predator), hence it changes its speed suddenly, giving rise to the propagation of a signal across the whole flock, which turns into a collective escaping maneuver. After the signal has passed, each individual will eventually go back to its physiological cruising speed. It seems reasonable to expect that this happens without oscillating back and forth around the cruising speed, but also quite swiftly, in order to restore as quickly as possible the original dynamical state. If our theory is correct, such a sensible way to go back to normal is achieved at critical damping. However, we cannot exclude the possibility of overdamped or oscillatory recovery, and more realistically we expect the system to be close to critical damping. Experiments should detect whether inertial (second-order) terms are present in the dynamics. Whether or not the dynamics is critically damped, though, will require working out the different parameters, which with our current experimental resolution seems harder, but not necessarily hopeless. Experimental efforts in this direction are under way.

\section{ACKNOWLEDGMENTS}

This work was supported by IIT-Seed Artswarm and European Research Council Starting Grant 257126. A.C. thanks William Bialek for interesting discussions on the subject of speed waves.
[1] S. K. Ma, Modern Theory of Critical Phenomena, Advanced Book Classics (Perseus, New York, 2000).

[2] G. Parisi, Statistical Field Theory, Frontiers in Physics (Addison-Wesley, Redwood City, CA, 1988).

[3] J. Sethna, Statistical Mechanics: Entropy, Order Parameters, and Complexity, Oxford Master Series in Physics Vol. 14 (Oxford University Press, Oxford, 2006).

[4] D. J. Sumpter, Collective Animal Behavior (Princeton University Press, Princeton, 2010).

[5] T. Vicsek and A. Zafeiris, Phys. Rep. 517, 71 (2012).

[6] T. Mora and W. Bialek, J. Stat. Phys. 144, 268 (2011).

[7] W. Bialek, Rep. Prog. Phys. 81, 012601 (2017).

[8] E. B. Stear, Self-Organizing Systems (Springer-Verlag, Berlin, Heidelberg, 1987), pp. 351-397.

[9] C. R. Kube and H. Zhang, in Proceedings of the Second International Conference on Simulation of Adaptive Behavior, edited by J.-A. Meyer, H. L. Roitblat, and S. W. Wilson, $A$ Bradford Book (MIT Press, Cambridge, 1993), pp. 460-468.

[10] A. Jadbabaie, J. Lin, and A. S. Morse, IEEE Trans. Autom. Control 48, 988 (2003).

[11] N. E. Leonard, D. A. Paley, F. Lekien, R. Sepulchre, D. M. Fratantoni, and R. E. Davis, Proc. IEEE 95, 48 (2007).

[12] M. Nagy, Z. Akos, D. Biro, and T. Vicsek, Nature (London) 464, 890 (2010).

[13] D. H. Kelley and N. T. Ouellette, Sci. Rep. 3, 1073 (2013).

[14] F. Ginelli, F. Peruani, M.-H. Pillot, H. Chaté, G. Theraulaz, and R. Bon, Proc. Natl. Acad. Sci. USA 112, 12729 (2015).

[15] C. Dombrowski, L. Cisneros, S. Chatkaew, R. E. Goldstein, and J. O. Kessler, Phys. Rev. Lett. 93, 098103 (2004).

[16] H.-P. Zhang, A. Be'er, E.-L. Florin, and H. L. Swinney, Proc. Natl. Acad. Sci. USA 107, 13626 (2010).
[17] B. Szabo, G. J. Szöllösi, B. Gönci, Z. Jurányi, D. Selmeczi, and T. Vicsek, Phys. Rev. E 74, 061908 (2006).

[18] Y. Katz, K. Tunstrøm, C. C. Ioannou, C. Huepe, and I. D. Couzin, Proc. Natl. Acad. Sci. USA 108, 18720 (2011).

[19] A. Strandburg-Peshkin, C. R. Twomey, N. W. Bode, A. B. Kao, Y. Katz, C. C. Ioannou, S. B. Rosenthal, C. J. Torney, H. S. Wu, S. A. Levin et al., Curr. Biol. 23, R709 (2013).

[20] J. Buhl, D. J. Sumpter, I. D. Couzin, J. J. Hale, E. Despland, E. R. Miller, and S. J. Simpson, Science 312, 1402 (2006).

[21] S. Goss, S. Aron, J.-L. Deneubourg, and J. M. Pasteels, Naturwissenschaften 76, 579 (1989).

[22] R. Cont and J.-P. Bouchaud, Macroecon. Dyn, 4, 170 (2000).

[23] D. Helbing, P. Molnár, I. J. Farkas, and K. Bolay, Environ. Plan. B 28, 361 (2001).

[24] C. W. Reynolds, ACM SIGGRAPH Comput. Graph. 21, 25 (1987).

[25] S. Krasner, The Ubiquity of Chaos (American Association for the Advancement of Science, Washington, D.C., 1990).

[26] J. Toner and Y. Tu, Phys. Rev. E 58, 4828 (1998).

[27] C. K. Hemelrijk and H. Hildenbrandt, Interface Focus (2012), doi: 10.1098/rsfs.2012.0025.

[28] D. Sumpter, J. Buhl, D. Biro, and I. Couzin, Theory Biosci. 127, 177 (2008).

[29] A. Attanasi, A. Cavagna, L. Del Castello, I. Giardina, T. S. Grigera, A. Jelić, S. Melillo, L. Parisi, O. Pohl, E. Shen et al., Nat. Phys. 10, 691 (2014).

[30] A. Procaccini, A. Orlandi, A. Cavagna, I. Giardina, F. Zoratto, D. Santucci, F. Chiarotti, C. Hemelrijk, E. Alleva, G. Parisi, and C. Carere, Anim. Behav. 82, 759 (2011).

[31] C. K. Hemelrijk, L. van Zuidam, and H. Hildenbrandt, Behav. Ecol. Sociobiol. 69, 755 (2015). 
[32] G. Tkačik and W. Bialek, Annu. Rev. Condens. Matter Phys. 7, 89 (2016).

[33] T. Vicsek, A. Czirók, E. Ben-Jacob, I. Cohen, and O. Shochet, Phys. Rev. Lett. 75, 1226 (1995).

[34] H. Chaté, F. Ginelli, G. Grégoire, and F. Raynaud, Phys. Rev. E. Stat. Nonlin. Soft Matter Phys. 77, 046113 (2008).

[35] J. Toner and Y. Tu, Phys. Rev. Lett. 75, 4326 (1995).

[36] Y. Tu, J. Toner, and M. Ulm, Phys. Rev. Lett. 80, 4819 (1998).

[37] J. Toner, Y. Tu, and S. Ramaswamy, Ann. Phys. 318, 170 (2005).

[38] M. Marchetti, J. Joanny, S. Ramaswamy, T. Liverpool, J. Prost, M. Rao, and R. A. Simha, Rev. Mod. Phys. 85, 1143 (2013).

[39] A. P. Solon, J. Stenhammar, R. Wittkowski, M. Kardar, Y. Kafri, M. E. Cates, and J. Tailleur, Phys. Rev. Lett. 114, 198301 (2015).

[40] T. Ihle, Phys. Rev. E 88, 040303 (2013).

[41] E. Bertin, M. Droz, and G. Grégoire, Phys. Rev. E 74, 022101 (2006).

[42] E. Bertin, M. Droz, and G. Grégoire, J. Phys. A 42, 445001 (2009).

[43] W. Bialek, A. Cavagna, I. Giardina, T. Mora, O. Pohl, E. Silvestri, M. Viale, and A. M. Walczak, Proc. Natl. Acad. Sci. USA 111, 7212 (2014).

[44] C. K. Hemelrijk and H. Hildenbrandt, J. Stat. Phys. 158, 563 (2015).

[45] F. Peruani and L. G. Morelli, Phys. Rev. Lett. 99, 010602 (2007).

[46] C. Huepe, E. Ferrante, T. Wenseleers, and A. E. Turgut, J. Stat. Phys. 158, 549 (2015).

[47] V. I. Smirnov, A Course of Higher Mathematics: Vol. 2, Advanced Calculus (Pergamon Press, New York, 1964).

[48] A. G. Webster and S. J. Plimpton, Partial Differential Equations of Mathematical Physics (Courier Dover Publications, New York, 2016).

[49] P. C. Magnusson, A. Weisshaar, V. K. Tripathi, and G. C. Alexander, Transmission Lines and Wave Propagation (CRC Press, Boca Raton, FL, 2000).

[50] A. Cavagna, I. Giardina, and T. S. Grigera, Phys. Rep. 728, 1 (2018).

[51] M. Aldana and C. Huepe, J. Stat. Phys. 112, 135 (2003).
[52] H. Chaté, F. Ginelli, G. Grégoire, F. Peruani, and F. Raynaud, Eur. Phys. J. B 64, 451 (2008).

[53] P. Romanczuk and L. Schimansky-Geier, Ecol. Compl. 10, 83 (2012).

[54] F. Peruani, A. Deutsch, and M. Bär, Eur. Phys. J.: Spec. Top. 157, 111 (2008).

[55] R. Grossmann, L. Schimansky-Geier, and P. Romanczuk, New J. Phys. 14, 073033 (2012).

[56] T. Ihle, Phys. Rev. E 83, 030901 (2011).

[57] M. Nagy, I. Daruka, and T. Vicsek, Physica A 373, 445 (2007).

[58] F. Ginelli and H. Chaté, Phys. Rev. Lett. 105, 168103 (2010).

[59] Y.-L. Chou, R. Wolfe, and T. Ihle, Phys. Rev. E 86, 021120 (2012).

[60] A. Peshkov, S. Ngo, E. Bertin, H. Chaté, and F. Ginelli, Phys. Rev. Lett. 109, 098101 (2012).

[61] R. Zwanzig, Nonequilibrium Statistical Mechanics (Oxford University Press, Oxford, 2001).

[62] É. Fodor, C. Nardini, M. E. Cates, J. Tailleur, P. Visco, and F. van Wijland, Phys. Rev. Lett. 117, 038103 (2016).

[63] D. Loi, S. Mossa, and L. F. Cugliandolo, Phys. Rev. E 77, 051111 (2008).

[64] U. M. B. Marconi and C. Maggi, Soft Matter 11, 8768 (2015).

[65] T. Mora, A. M. Walczak, L. Del Castello, F. Ginelli, S. Melillo, L. Parisi, M. Viale, A. Cavagna, and I. Giardina, Nat. Phys. 12, 1153 (2016).

[66] A. Cavagna, I. Giardina, T. S. Grigera, A. Jelic, D. Levine, S. Ramaswamy, and M. Viale, Phys. Rev. Lett. 114, 218101 (2015).

[67] K. Huang, Introduction to Statistical Physics (CRC Press, Boca Raton, 2009).

[68] W. A. Strauss, Partial Differential Equations: An Introduction (Wiley, New York, 1992).

[69] J. R. Taylor, Classical Mechanics (University Science Books, Sausalito, California, 2005).

[70] A. Cavagna, D. Conti, I. Giardina, T. S. Grigera, S. Melillo, and M. Viale, Phys. Biol. 13, 065001 (2016).

[71] A. Cavagna, D. Conti, C. Creato, L. Del Castello, I. Giardina, T. S. Grigera, S. Melillo, L. Parisi, and M. Viale, Nat. Phys. 13, 914 (2017).

[72] D. A. Paley and A. K. Baharani, American Control Conference (ACC), 2010 (IEEE, 2010), pp. 4628-4633. 